\title{
A digital road map analogy of the relationship between neuroscience and educational research
}

\author{
Elsbeth Stern • Michael Schneider
}

Accepted: 28 August 2010/Published online: 17 September 2010

(C) FIZ Karlsruhe 2010

\section{Introduction}

At a recent meeting about neuroscience and education, an experienced teacher stated: "Over the last decades, in many countries school education has seen one reform after another. None of them really worked. Doesn't this prove that we need neuroscience to make school instruction work?" While we politely smiled and nodded, we could not help noticing the irony in this proposal. It is certainly true that school education has seen many reforms come and go. Each of them was motivated by dissatisfaction with existing approaches and by the hope for a better future. However, in the end, dissatisfaction and hope alone are poor fundaments for educational reform. This young teacher expressed the same dissatisfaction with the status quo and the same vague hope for a better future when he argued for the necessity of a neuroscience approach to education. Did he ever ask himself if educational neuroscience might simply be yet another "scientific revolution", in a series of many such "revolutions", each leading to a brief period of disappointment, to be followed by yet another such "revolution" in 10 or 20 years?

Scientists who believe that neuroscience should have a strong impact on education often claim that educational science is a soft science, which gives fuzzy, complicated answers that are based mainly on opinions, while cognitive neuroscience is a hard science, which gives clear and precise answers based on unambiguous empirical evidence. They hope that cognitive neuroscience will yield clear and

E. Stern · M. Schneider $(\bowtie)$

Institute for Behavioral Sciences, ETH Zurich,

Universitaetsstrasse 41, 8092 Zurich, Switzerland

e-mail: schneider@ifv.gess.ethz.ch precise answers to the question "What is effective instruction and how can it be implemented?" So far, educational science has not provided answers to everyone's satisfaction.

Opponents of this view paint a very different picture. They emphasize that neuroscience deals with biological processes in the central nervous system, while educational science deals with institutionalized learning of cultural competencies that can only be assessed by behavioral means, such as tests and conversations. As yet, brain imaging methods rarely reveal innovative insights into learning in real-world contexts. They have many technical and logistic constraints because the research must be done under restricted conditions in the laboratory rather than in the classroom. To avoid noisy data caused by body movements, participants must indicate their answers by pushing buttons, which limits social interaction. Further, because of the high measurement error, brain activation data must be averaged over numerous trials to gain an acceptable signal-to-noise ratio. When participants solve any task, almost their whole brain is active. Thus, activation differences between different experimental conditions can only be interpreted when the two experimental conditions are very similar to each other and differ only in one or two basic cognitive processes they evoke. This is hardly possible in real-life contexts with ecologically valid, complex learning materials.

Given these two divergent positions, why should we have a special issue entitled "Cognitive neuroscience and mathematics education research"? We believe that mathematics is a particularly suitable starting point for addressing scientific issues related to the interaction between the brain and learning. We will use an analogy to describe how neuroscience and educational research can complement each other. 


\section{The digital roadmap analogy}

Digital road maps such as Google Maps, for example, have become increasingly popular over the last 10 years because of the many advantages they offer. One advantage is the zoom function. When a digital map is first opened, it is usually on an intermediate zoom level. At this level, it is easy to see which streets lead from point A to point B in a city, but the level of detail is too low to identify individual buildings and too high to show the location of the respective city in its country. In some situations, for example, when looking for the entrance of a park, it is therefore advisable to increase the zoom level.

In our opinion, the relation between a map on a lower zoom level and the same map on a higher zoom level is an excellent analogy of the relation between educational science and neuroscience. In particular, it illustrates the following four points. First, cognitive neuroscience is generally relevant to educational science. They both investigate human learning, even though they look very different from each other at first glance, just as the same map looks very different when viewed at different zoom levels.

Second, whether neuroscience is more useful or educational science is more useful depends on the specific purpose at hand, just as the optimal level of resolution of a map depends on what the map viewer is looking for. Classical behavioral educational science corresponds to an intermediate zoom level, which is useful in many situations, for example, when teachers really need to figure out how to get their students from point $\mathrm{A}$ to point $\mathrm{B}$ in terms of their behaviorally and verbally expressed thoughts and feelings. Educators only need to worry about the specific brain mechanisms underlying this behavior in certain exceptional cases. In these cases, they can zoom in on these specific processes. However, zooming to a high resolution always entails the danger of losing orientation in too many details. Therefore, we think that neuroscience, when necessary at all, should be used only by educational researchers with a working knowledge in this domain and not by actual teachers. While it might on some days be possible to explain everything that happens in a classroom in terms of what the nerve cells, muscles, and sensorial cells of all the people involved are doing, such a description would be far too complex and lengthy to be useful for any practical pedagogical purpose. In some situations, it might even be useful to view learning processes at an even lower level of detail than that of educational science and zoom out to the level of the historical, economical, or political contexts of schooling.

Third, a map is always an abstraction and simplification of the reality it represents, just as any empirical science is always an abstraction and simplification of its subject.
People who criticize educational science for being fuzzy should remember that the brain has more than $15,000,000,000$ nerve cells, and each of them has up to 10,000 inter-connections with other brain cells. Within each nerve cell and each connection between them, a multitude of chemical and physical processes (i.e., flow of electrical charges) run in parallel and interact. In comparison, an electroencephalogram (EEG) is usually only measured by up to 128 electrodes, and functional magnetic resonance imaging (fMRI) often yields far less than $1,000,000$ three-dimensional picture points. Thus, while brain imaging methods might seem to be more precise than behavioral methods in some cases, brain imaging methods do by no means show the brain "as it really is". Their results are filtered, aggregated, and processed. Which methods are adequate for filtering, aggregating, and processing brain imaging data under what circumstances is a matter of conventions, ongoing negotiations, and decisions of the scientific community.

In terms of our analogy, cognitive neuroscience investigates learning on a gradually higher zoom level than educational science, but there are many even higher zoom levels that deal with the neuronal, chemical, and physical underpinnings of brain activation patterns.

Fourth, when a person jumps from a very low to a very high resolution on a digital map orientation, it is generally lost because the leap makes it hard to determine the exact location of the small section that was visible before on the larger map that is displayed afterward. For this reason, most people zoom successively from a low zoom level through increasingly higher levels to the highest desired level in the end. This concept is also useful when thinking about how phenomena from the world of educational science connect to underlying brain mechanisms. Behavioral cognitive science is an indispensable intermediate zoom level, which contributes to a better understanding of concrete connections between knowledge construction and its neural correlates. This has also been pointed out by James T. Bruer in his seminal article "Education and the Brain: A Bridge Too Far".

\section{The landscape of research on mathematics learning}

If we see mathematics learning as a landscape that can be viewed from different zoom levels, then what is the basic structure of this landscape? In the following paragraphs, we give a short summary.

Research questions related to working memory functions, reasoning, and transfer are often addressed by using mathematical problems because these problems allow systematic variations in their complexity as well as in their degree of abstraction. Insights into the constraints of the 
human mind, such as situated cognition or cognitive load, have been obtained in studies using mathematical tasks. Besides the universal perspective, research based on mathematical tasks has also contributed to the developmental and differential perspective of the human mind. There is overwhelming evidence from infant research that humans are endowed with basic numerical abilities that help them to organize incoming information from the world and which provide the foundations for learning to count. Yet, despite these universal resources, the wide range of individual differences in mathematical abilities is already apparent in young children.

The saying "God created the natural numbers; everything else is the work of man" highlights this characteristic of mathematics. On the basis of innate cognitive resources, different groups of human beings have developed mathematical symbol systems, which in turn have given rise to specific mathematical disciplines. To meet the demands of academic mathematics, one must understand the dual nature of the symbols, that is, although the symbols can represent events and situations of the external world (signifier function), they are also meaningful in themselves (signified function). Cultural mathematics requires the understanding that numbers are not only used for counting and denomination of set sizes, but also for describing the relations between sets. This is the case with rational numbers, for instance, which, in contrast to natural numbers, neither have a determined antecessor or follower. Understanding abstract mathematical concepts mandates separation of mathematical symbols from concrete contexts. One must deal with symbols having no concrete, real-world referential meaning. The algebra system, for example, allows extensive manipulation of relationships among variables within a well-specified system without referring to the external world. The principles underlying the numbersymbol system allow the development of new concepts because numbers and symbols of operations are meaningful even if they do not refer to real-world situations.

Individual differences in mathematical competencies are tremendous. At one end of the spectrum, a minority of children cannot comprehend even very basic numerical problems, indicating brain dysfunction. At the other end of the spectrum are the mathematically gifted. Even at very young ages, they have an intuitive understanding of numbers, their magnitudes, relationships, and how they are affected by operations. Mathematical giftedness is closely related to, but not identical with, general intelligence. What kind of domain-specific and domain general abilities have to accrue to provide the preconditions for mathematical giftedness?

While the vast majority of students acquire basic numerical competencies without effort, most struggle with advanced mathematics. Even among the students who meet classroom achievement goals, mixed to negative feelings regarding advanced mathematics are quite common. Mathematics is a major source of school anxiety, which makes it appropriate for research on emotions and learning. Given the progress made by brain imaging in displaying neural correlates of emotions, research on learning mathematics can shed light on the interaction between cognition and emotion on a behavioral as well as on a neural basis.

There is one more characteristic of mathematical cognition that makes the field particularly appropriate for brain imaging that is worthy of mention: cognitive models of knowledge representation and transformation are more advanced than in any other field. The interaction between verbal, numerical, and visual-spatial knowledge representation has been addressed in many studies. The same holds true for how procedural knowledge emerges from declarative knowledge and how a network of number facts arises out of frequent strategy use.

\section{The ten contributions to the special issue}

This special issue presents ten papers, which address questions about neural correlates of mathematical competencies in different age groups and at different achievement levels. The mathematical problems presented in the brain imaging studies cover the broad range of underlying knowledge types and representational resources. In the first contribution, entitled "Developmental cognitive neuroscience of arithmetic: Implications for learning and academic achievement", Menon gives an overview of studies that attempt to localize brain activity, while students solve mathematical problems of different formats. Butterworth and Laurillard strengthen the claim that inflexibility in representational transformations is a major obstacle for a group of low performers in the second paper "Low numeracy and dyscalculia: Identification and intervention", by presenting promising results of an intervention study. The third paper, written by a research group from Munich (Obersteiner, Dresler, Reiss, Vogel, Pekrun, \& Fallgatter) takes into consideration a broad range of students when investigating arithmetic problems. In their paper "Bringing brain imaging to the school to assess arithmetic problem solving: Chances and limitations in combining educational and neuroscientific research", they discuss the pros and cons of the NIRS method, which in principle has potential for classroom use.

Paper number four by a research group from the Humboldt University in Berlin (Bornemann, Foth, Horn, Ries, Warmuth, Wartenburger, \& van der Meer) investigates how general intelligence comes into play in mathematical 
cognition in the article "Mathematical cognition: Individual differences in resource allocation." The fifth paper by Zago, Petit, Mellet, Mazoyer, and Tzourio-Mazoyer, "Neural correlates of counting large numerosity", presents brainbased evidence showing that different cognitive resources contribute to the processing of small and large numbers. Landgraf, van der Meer, and Krueger emphasize in the sixth contribution that there is more to investigating neural correlates than just brain imaging. In their article "Cognitive resource allocation for neuronal activity underlying mathematical cognition: A multi-method study", they prove the value of eye tracking methods for better understanding learning processes.

The topic of how different representational formats are processed in advanced mathematical reasoning and how the representations map on the brain is the subject of papers 7 and 8. Paper 7 is entitled "Computing solutions to algebraic problems using a symbolic versus a schematic strategy" by Lee, Yeong, Venkatraman, Graham, Chee, and Ng. Paper 8 is entitled "Evidence from cognitive neuroscience for the role of graphical and algebraic representations in understanding function" by Thomas, Wilson, Corballis, Lim, and Yoon. In the ninth paper, "Overcoming intuitive interference in mathematics: Insights from behavioral, brain imaging and intervention studies", Stavy and Babai investigate how the brain manages incongruent information in mathematical tasks. The final paper, "Long-term characteristics of analogical processing in high-school students with high fluid intelligence: An fMRI study", by Preusse, van der Meer, Ullwer, Brucks, Krueger, and Wartenburger explores how general intelligence in the long run complements mathematical abilities.

Altogether, this special issue should be viewed as a stepping stone toward further research on the interaction between mathematical problem solving and its neural correlates, in particular, and between the mind and the brain, in general. 